\title{
microRNA let-7i-5p aggravates kidney fibrosis via targeting GALNT1
}

\author{
Chen-Min Sun, Wen-Yi Zhang, Shu-Yan Wang, Gang Qian, Dong-Liang Pei and Guang-Ming \\ Zhang
}

Department of Anesthesiology, Tongren Hospital, Shanghai Jiao Tong University School of Medicine, Shanghai, China

\begin{abstract}
Renal fibrosis poses critical health problem. We aimed to investigate role of let-7i-5p in renal fibrosis. In silico reproduction of Mouse Kidney FibrOmics browser was used to identify potential target of let-7i-5p. In vivo validation was conducted in C57BL/6 mice with unilateral ureteral obstruction (UUO) and folic acid (FA) induction. In vitro validation was performed in transforming growth factor (TGF)- $\beta 1$-treated HK-2 cells. Mimics and inhibitors of let-7i-5p, and target gene polypeptide $\mathrm{N}$-acetylgalactosaminyltransferase 1 (GALNT1) were monitored by RT-PCR and Western blotting. Fibrosis markers, injury markers, and house-keeping genes were evaluated. Levels of interleukin (IL)-6, IL-1 $\beta$, and tumor necrosis factor (TNF)- $\alpha$ in serum and media were measured by ELISA. In silico analysis showed gradual increase of let-7i-5p and decrease of GALNT1 over time and the combination was validated both in mouse and human miR-gene target prediction databases. Expression of GALNT1 decreased while expression of let-7i-5p increased in renal tissues of both UUO and FA mice. Serum IL-6, IL-1 $\beta$, and TNF- $\alpha$ levels were elevated in vivo. In vitro models revealed negative correlation between expression levels of let-7i-5p and GALNT1. Overexpression of let-7i-5p inhibited GALNT1 expression and reduced release of inflammatory factors. In conclusion, overexpression of GALNT1 may combat the inflammation induced by let-7i-5p.
\end{abstract}

Key words: Renal fibrosis - GALNT1 - let-7i-5p - Inflammation

Abbreviations: FA, folic acid; GALNT1, polypeptide N-acetylgalactosaminyltransferase 1; IL, interleukin; TGF- $\beta 1$, transforming growth factor- $\beta 1$; UUO, unilateral ureteral obstruction.

\section{Introduction}

Renal fibrosis is a pathophysiological change. It is a gradual process in which the function of the kidney's changes from health to injury, to damage, until loss of function (Sun et al. 2016). The kidney is stimulated by various pathogenic factors such as trauma, infection, inflammation, blood circulation disorders, and immune response. Its inherent cells are damaged, and a large amount of collagen deposition and accumulation occurs in the later stage, causing the renal parenchyma to gradually harden and form scars until the complete loss of organ function (Nogueira et al. 2017). The process of fibrosis and sclerosis inherent in the kidney is

Correspondence to: Guang-Ming Zhang, Department of Anesthesiology, Tongren Hospital, Shanghai Jiao Tong University School of Medicine, Shanghai 200336, China

E-mail: dr_anesthesiology@163.com also the process of renal fibrosis. Renal fibrosis is characterized by abnormal deposition of extracellular matrix (ECM) (Nastase et al. 2018).

The primary and secondary causes of kidney disease, through the damage to the kidney, activate capillary endothelial cells in the glomerulus and attract the infiltration of inflammatory cells in the blood, and at the same time release nephrotoxic inflammatory mediators, causing intrarenal inflammatory response. In addition, under the action of a series of nephrotoxic factors, the original functional cells of the kidney were damaged and the phenotype changed. The phenotype-changed intrinsic cells secreted a series of nephrotoxic cytokines and growth factors (Sun et al. 2017). These nephrotoxic cytokines and growth factors in turn activate fibroblasts in the renal mesenchyme to transform into myofibroblasts. Myofibroblasts are the key cells that cause kidney fibrosis. The collagen fibers they secrete are difficult to degrade, which leads to the accumulation and deposition 
of a large amount of extracellular matrix collagen, which destroys the kidney tissue structure. Eventually scar tissue is formed. Renal function also shows a progressive decline, leading to the destruction of all or most of the nephrons and the complete or almost complete loss of function. Clinically manifests as end-stage renal failure (uremia).

microRNAs (miRs) are a class of non-coding singlestranded RNA molecules encoded by endogenous genes with a length of about 22 nucleotides. They can regulate gene expression by inhibiting the translation of target mRNA or reducing the stability of target mRNA (Loboda et al. 2016). Since miRs was listed by the Science Magazine as one of the top ten scientific and technological breakthroughs in the world in 2002, miRs have gradually become a hot spot in life science research. Renal fibrosis is a common pathological process from the development of chronic kidney disease to the late stage caused by various etiologies. It is accompanied by a progressive decrease in renal function. Its pathological morphology is characterized by the gradual decrease of renal parenchymal cells, the proliferation of interstitial cells and the accumulation of extracellular matrix. So far, the mechanism of renal fibrosis is complex, and some molecular mechanisms remain to be elucidated. The discovery of miRs opens a new perspective for the research of the mechanism of renal fibrosis, and in-depth study of the role of miRs in renal fibrosis will help discovery of new drug targets for renal fibrosis and identification of prognostic biomarkers (Lv et al. 2018).

In the current study, we performed in silico analysis of multi-omics in both folic acid (FA) and unilateral ureteral obstruction (UUO) that respectively represent the reversible and irreversible stage of renal fibrosis to identify potential $\mathrm{miR}$ and target genes that play a role in the pathophysiology of renal fibrosis.

\section{Materials and Methods}

\section{In silico analysis}

Candidate miRs and genes were selected using the Mouse Kidney FibrOmics online database. The platform was a comprehensive and combined multi-omics dataset (proteomics, mRNA and small RNA transcriptomics) of fibrotic kidneys that is searchable through a user-friendly web application: http://hbcreports.med.harvard.edu/fmm/. Two commonly used mouse models were utilized in the dataset, a reversible chemical-induced injury model (FA induced nephropathy) and an irreversible surgically-induced fibrosis model (UUO). mRNA and small RNA sequencing, as well as 10plex tandem mass tag (TMT) proteomics were performed with kidney samples from different time points over the course of fibrosis development were included in the dataset. Values were $\mathrm{z}$-scores of the $\log 2$ normalized abundance for each molecule. Predicted interactions between miRNA and candidate target gene was according to Targetscan platform (Pavkovic et al. 2019). Our strategy to select candidate miRgene pair denoted the pairs conforming to all of the following criteria: a) alteration of miR showing a consistent trend of increase in both FA and UUO models; b) target gene of the corresponding miR showing a correlation coefficient of -1.0 ; c) target gene showing a consistent trend of decrease at both mRNA and protein levels. Candidate pairs were selectively tested in HK-2 cells using PCR to examine expression of target gene in response to miR addition and only the pairs with strongest inhibitory effects were selected.

\section{Mouse models}

Male BALC/c mice were obtained from Shanghai Silaike Experiment Animal Co, Ltd. All animal studies were conducted in accordance with the Ethical Guide for the Care and Use of Laboratory Animals. Mice were housed in groups of six on a 12-h light/dark cycle with access to food and water ad libitum. At the age of 8-10 weeks they entered the experiment. FA was prepared at $25 \mathrm{mg} / \mathrm{ml}$ in $0.3 \mathrm{M}$ sodium bicarbonate. Mice received a single dose of $250 \mathrm{mg} / \mathrm{kg}$ via intraperitoneal injection. Before the injection and 7 days later, mice were sacrificed; kidneys were removed and immediately snap frozen in liquid nitrogen. For UUO group, mice were anesthetized with $(10 \mu \mathrm{l}$ ketamine $+1 \mu \mathrm{l}$ xylazine $) / \mathrm{g}$ body weight. The abdominal cavity was opened and the left ureter was ligated twice. Sham operations were done without ureteral ligation. On day 3 and day 14, mice were sacrificed to collect renal tissues and blood samples.

\section{Cell culture and transfection}

HK-2 human renal tubular epithelial cell line was obtained from cell bank of Chinese Academy of Science (Shanghai, China) and was cultured in DMEM supplemented with 10\% FBS. Cells were treated with transforming growth factor (TGF)- $\beta 1$ ( $10 \mathrm{ng} / \mathrm{ml})$ for $48 \mathrm{~h}$ to induce fibrosis. To modulate let-7i-5p expression levels, HK-2 cells were transfected with $\mathrm{miR}$ mimic or inhibitor, both synthesized by Ribobio (Guangzhou, China). Adenovirus (Av)-delivered overexpression of human polypeptide $\mathrm{N}$-acetylgalactosaminyltransferase 1 (GALNT1) was constructed with cDNA clone obtained from Origene. All transfections were conducted using Lipofectamine 2000 system according to manufacturer instructions. The cells were harvested, $48 \mathrm{~h}$ after transfection, for further studies.

\section{Real-time PCR}

Although there were various markers for fibrosis and injury of kidney, we referenced the multi-omics data that comprised 
Table 1. Primers used in the study (5' to $\left.3^{\prime}\right)$

\begin{tabular}{lll}
\hline Gene & Forward & Reverse \\
\hline Acta2 & CCCAACTGGGACCACATGG & TACATGCGGGGGACATTGAAG \\
Colla1 & GCTCCTCTTAGGGGCCACT & CCACGTCTCACCATTGGGG \\
Col1A1 & GAGGGCCAAGACGAAGACATC & CAGATCACGTCATCGCACAAC \\
Fn1 & ATGTGGACCCCTCCTGATAGT & GCCCAGTGATTTCAGCAAAGG \\
Clu & GCTGCTGATCTGGGACAATG & ACCTACTCCCTTGAGTGGACA \\
Havcr 1 & ACATATCGTGGAATCACAACGAC & ACTGCTCTTCTGATAGGTGACA \\
Lcn2 & TGGCCCTGAGTGTCATGTG & CTCTTGTAGCTCATAGATGGTGC \\
Actb & GTGACGTTGACATCCGTAAAGA & GCCGGACTCATCGTACTCC \\
B2m & TTCTGGTGCTTGTCTCACTGA & CAGTATGTTCGGCTTCCCATTC \\
Gapdh & AGGTCGGTGTGAACGGATTTG & GGGGTCGTTGATGGCAACA \\
GAPDH & GGAGCGAGATCCCTCCAAAAT & GGCTGTTGTCATACTTCTCATGG \\
Gusb & CCGACCTCTCGAACAACCG & GCTTCCCGTTCATACCACACC \\
Hprt1 & CCTGGCGTCGTGATTAGTGAT & AGACGTTCAGTCCTGTCCATAA \\
IL-6 & AAGAGCCGGAAATCCACGAAA & GTGCGAGGTCTGGGATATTGC \\
IL-1ß & GAAATGCCACCTTTTGACAGTG & TGGATGCTCTCATCAGGACAG \\
TNF- $\alpha$ & CAGGCGGTGCCTATGTCTC & CGATCACCCCGAAGTTCAGTAG \\
Galnt1 & TCCTGCTGCTTTACTTCAGTG & TGACGACTGGTTTCCCCATTT \\
GALNT1 & CACCTCCTTGATTTGGGTACTC & AGGAATGACGACTGGTTCCC \\
U6 & CTCGCTTCGGCAGCACA & AACGCTTCACGAATTTGCGT \\
let-7i & Reverse transcription: AACGCTTGACGAATTTGCGC & \\
\cline { 2 - 3 } & TAGTACTGCGCAAGCTACTGC & GTGCAGGGTCCGAGGT \\
\hline Reverse transcription: CTCAACTGGTGTCGTGGAGTCGGCAATTCAGTTGAGAGCACAAA
\end{tabular}

Note: Gene names with upper case indicate human origin and with lower case indicate mouse origin.

of the FibrOmic dataset and selected the markers used in this particular dataset to maintain consistency (Pavkovic et al. 2019). Total RNA was extracted by Trizol and converted to cDNA. Quantitative real-time PCR was carried out using SYBR ExScript real-time PCR on ABI 7500 real-time PCR, according to manufacturer's protocol. Glyceraldehyde3-phosphate dehydrogenase (GAPDH) was used as endogenous control. Expression of let-7i-5p was measured using Hairpin-it miRNAs qPCR Quantitation Kit. U6 small nuclear RNA was used as an internal control for miR. Primers used were listed in Table 1.

\section{Western blotting}

Protein samples $(20 \mu \mathrm{g} / \mathrm{lane})$ were separated by electrophoresis on $10 \%$ polyacrylamide gel and then transferred onto polyvinylidene fluoride (PVDF) membranes. The membranes were blocked with 5\% non-fat milk and incubated with primary antibodies overnight at $4^{\circ} \mathrm{C}$. The next day, membranes were incubated with horseradish peroxidase (HRP)-conjugated secondary anti- body at room temperature. The blots were visualized with enhanced chemiluminescence method using ECL kit. Primary antibodies used were: Polyclonal GALNT1 Antibody (WB) LS-C307642 (LSBio) at 1:500 and Anti-GAPDH antibody (ab9484) (Abcam) at 1:1000.

\section{Measurement of inflammatory factors}

Inflammatory factors of interleukin (IL)- $1 \beta$, IL-6, and tumor necrosis factor $\alpha$ (TNF- $\alpha$ ) were detected in blood samples and cell culture supernatants. BD OptEIA and ELISA kits for mouse cytokines and human cytokines were used, respectively.

\section{Statistical analysis}

In vitro experiments were performed in triplicates. For comparisons in more than 2 factors in more than 2 groups, the two-way analysis of variance (ANOVA), followed by post-hoc tests (Bonferroni) was used. For the rest of experiments, one-way ANOVA with Tukey's multiple comparisons test was used. The significance was designated as $p<0.05$.

\section{Results}

To investigate possible culprit miRs and target genes in renal fibrosis, we first performed in silico analysis of a comprehensive and robust dataset of FA- and UUO-induced mouse models. Based on pilot assays, we found consistent upregulation of mmu-let-7i-5p in both FA and UUO models throughout 7 days of establishment with decline of the miR on day 14 when 


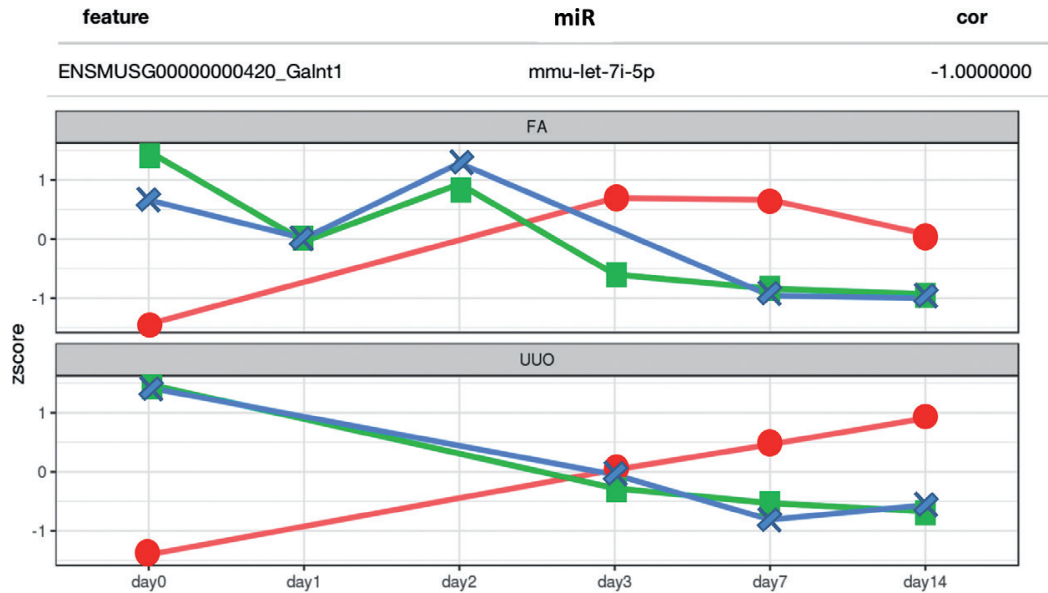

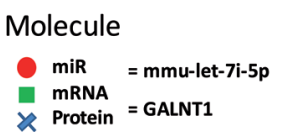

Figure 1. In silico analysis of Mouse Kidney FibrOmics dataset showing micro-RNA (miR) expression and potential target gene expression indicated by "feature" and protein levels in FAand UUO-induced renal fibrosis mouse models. Cor, correlation coefficient; FA, folic acid; UUO, unilateral ureteral obstruction.
mRNA and protein levels of target gene declined (Fig. 1). TargetScan platform showed the predicted target gene to be Polypeptide N-acetylgalactosaminyltransferase 1 (GALNT1) with a prediction correlation of -1.00 . The tendency of change of miR let-7i-5p and mRNA level of GALNT1 showed a corresponding pattern of $\mathrm{miR}$-gene regulation. Of note, the pattern showed consistency in both FA and UUO models.

We then conducted in vivo validation of the findings in silico (Fig. 2A). For both FA and UUO model, we used a 7-day treatment course according to findings in silico. To examine accuracy to modeling, we found that most injury markers (Fig. 2B) and fibrosis markers (Fig. 2C) were significantly elevated following model establishment. The effect of UUO or FA on both markers was significant $(\mathrm{F}(6,60)=13.72, p<0.001)$. Effect of UUO on Clu $(p<0.001)$, Havcr $1(p<0.001)$ and Lcn $2(p<0.001)$ was significant, respectively. Effect of FA on Clu $(p=0.1439)$ or Havcrl $(p=0.4035)$ was not significant, respectively. Effect of FA on Lcn2 was significant $(p<0.001)$. As expected, reversible model using FA showed milder alteration of fibrosis markers which represented irreversible stage of clinical course (Fig. 2B,C). The effect of UUO or FA on both markers was significant $(\mathrm{F}(6,60)=63.42$,
A
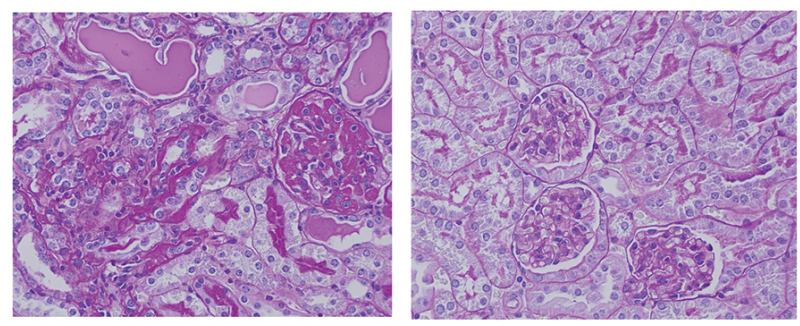

C

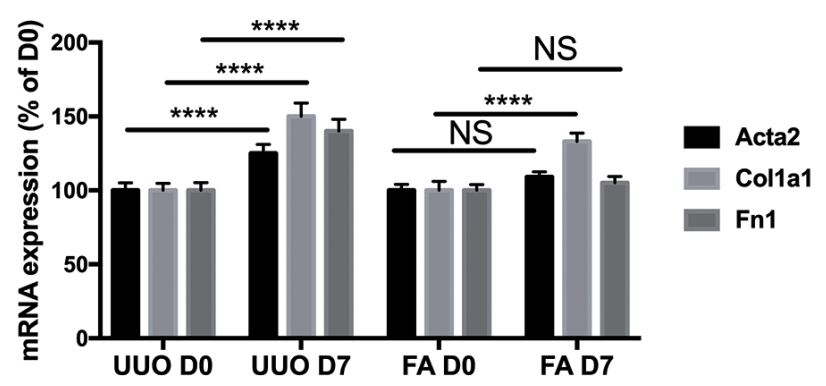

B

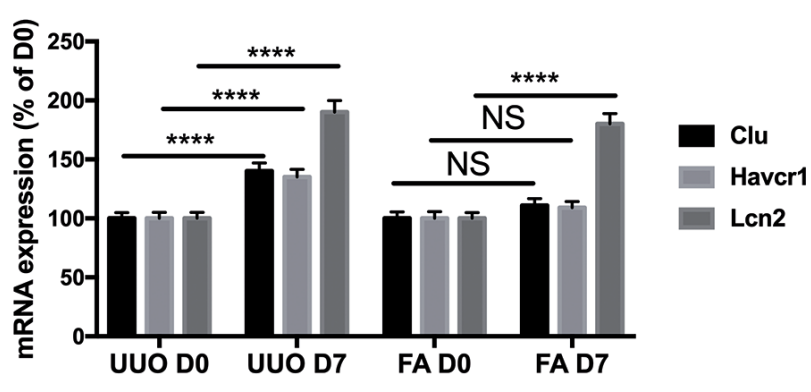

D

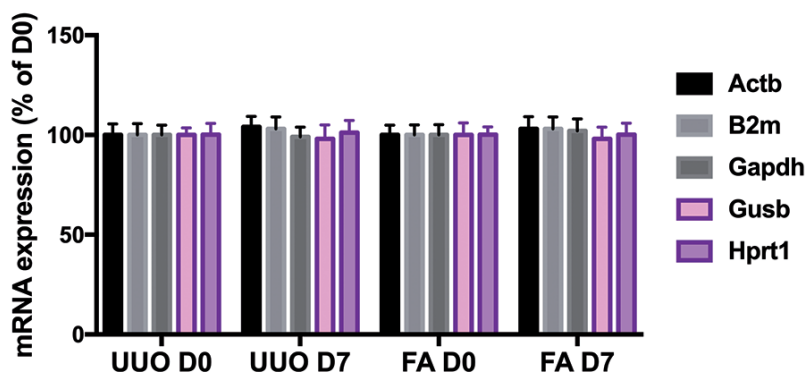

Figure 2. Mouse models of kidney fibrosis of FA and UUO. A. Representative Picrosirius-staining image of fibrosis (left) and control (right). Expressions of fibrosis markers (B), injury markers (C) and house-keeping genes (D) in ipsilateral kidney tissue. ${ }^{* *} p<0.001$, ${ }_{* * * *} p<0.0001$. D0, day 0; D7, day 7; NS, not significant. 
A

IL-6

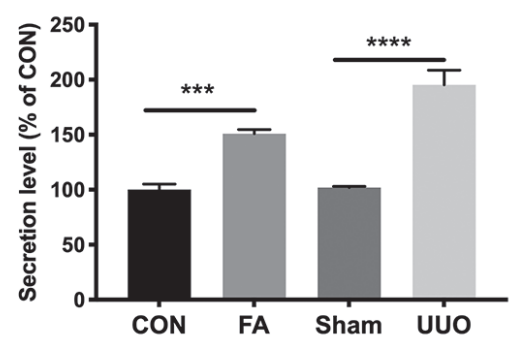

B

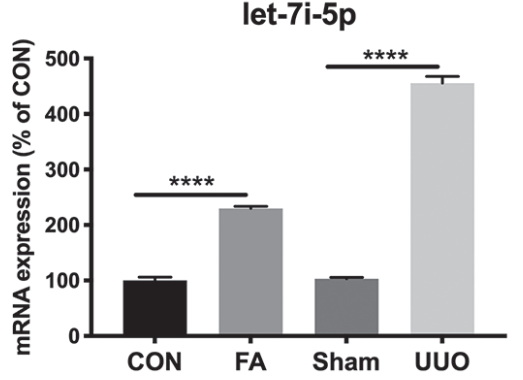

IL-1 $\beta$

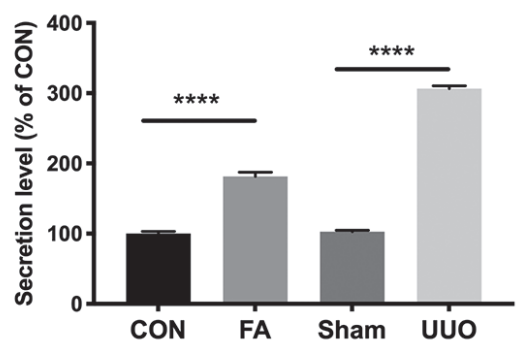

GALNT1

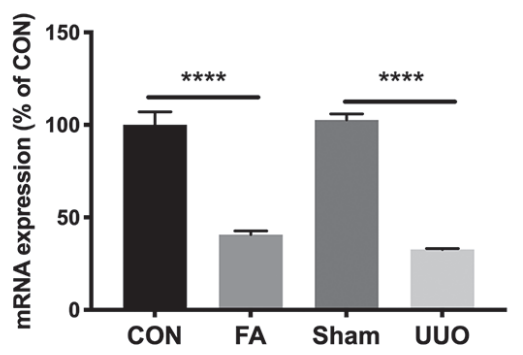

TNF- $\alpha$

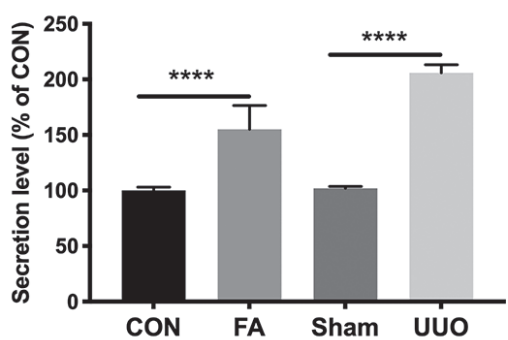

Figure 3. A. Serum inflammatory markers including IL-6, IL-1ß, and TNF- $\alpha$. B. mRNA expression of let-7i-5p and GALNT1, and protein level of GALNT1 of mouse models of kidney fibrosis of FA and UUO. ${ }^{* *} p<0.001$; ${ }^{* * *} p<0.0001$. CON, control; FA, folic acid; GALNT1, polypeptide N-acetylgalactosaminyltransferase 1; UUO, unilateral ureteral obstruction.

$p<0.001)$. Effect of UUO on Acta2 $(p<0.001)$, Colla1 $(p<$ $0.001)$ and Fn1 $(p<0.001)$ was significant, respectively. Effect of FA on Acta2 $(p=0.2338)$ or Fn1 $(p=0.1218)$ was not significant, respectively. Effect of FA on Collal was significant $(p<$ 0.001). In comparison, house-keeping genes showed consistent expression levels throughout treatment course (Fig. 2D). Effect of neither UUO nor FA was significant on house-keeping genes $(\mathrm{F}(12,100)=0.4079, p=0.9575)$.
Also, inflammatory factors were significantly elevated in both FA and UUO mouse models (Fig. 3A). Ipsilateral kidneys were harvested on day 7 and test for mRNA expression of let-7i$5 p$ and GALNT1 showing significantly elevated let-7i-5p level and decreased GALNT1 level (Fig. 3B). Validation of protein showed corresponding protein change of GALNT1 (Fig. 3B).

To mimic physiology in human, we used TGF- 31 -induced HK-2 cell as model. We found TGF-ß1 significantly induced

Table 2. Post-hoc analysis of comparisons of parameters in HK2 cells shown in Figure 4

\begin{tabular}{lccc}
\hline \multicolumn{3}{c}{ Tukey's multiple comparisons test } \\
\cline { 2 - 4 } & \multicolumn{3}{c}{$p$ value } \\
\cline { 2 - 4 } & let-7i-5p & GALNT1 & Collagen I \\
\hline CON $v$ s. TGF- $\beta 1$ & $<0.0001$ & $<0.0001$ & $<0.0001$ \\
CON $v$ s. TGF- $\beta 1+$ miRCon & $<0.0001$ & $<0.0001$ & $<0.0001$ \\
CON $v$ s. TGF- $\beta 1+$ Let-Inh & 0.5078 & $<0.0001$ & 0.005 \\
CON $v$ s. Let-mimic & $<0.0001$ & $<0.0001$ & - \\
TGF- $\beta 1$ vs. TGF- $\beta 1+$ miRCon & 0.9986 & 0.7367 & 0.9892 \\
TGF- $\beta 1$ vs. TGF- $\beta 1+$ Let-Inh & $<0.0001$ & $<0.0001$ & $<0.0001$ \\
TGF- $\beta 1$ vs. Let-mimic & $<0.0001$ & 0.9026 & - \\
TGF- $\beta 1+$ miRCon $v$ s. TGF- $\beta 1+L e t-I n h$ & $<0.0001$ & $<0.0001$ & $<0.0001$ \\
TGF- $\beta 1+$ miRCon $v$ s. Let-mimic & $<0.0001$ & 0.293 & - \\
TGF- $\beta 1+$ Let-Inh $v s$. Let-mimic & $<0.0001$ & $<0.0001$ & - \\
\hline CON contol Let-Inh, let-71-5p & &
\end{tabular}

CON, control; Let-Inh, let-7i-5p inhibitor; Let-mimic, let-7i-5p mimic; miRCon, control micro-RNA. 

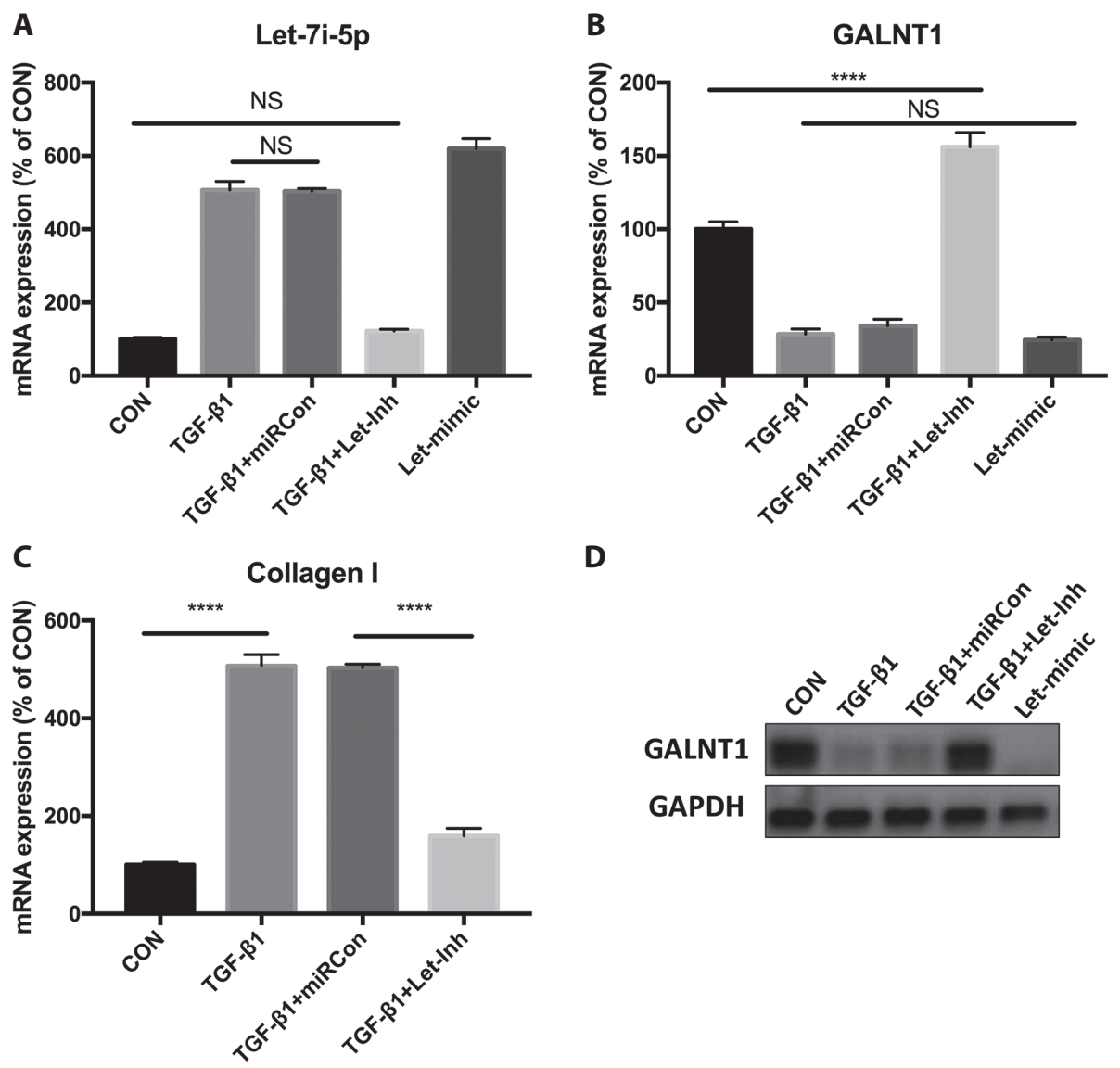

D

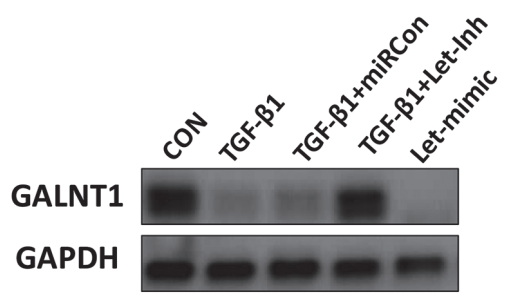

Figure 4. Overexpression of let-7i$5 \mathrm{p}$ inhibited GALNT1 expression and Collagen I expression in HK-2 cells treated with TGF- $\beta 1$. Relative expression of let-7i-5p (A), GALNT1 (B), Collagen I (C). D. Protein levels of GALNT1. ${ }^{* * * *} p<0.0001$. upregulation of let-7i-5p, which showed comparable level to let-7i-5p mimic (Fig. 4A). Level of let-7i-5p was significant different among groups $(\mathrm{F}(4,10)=627.6, p<0.001)$. A detailed comparison between groups were shown in Table 2. Expression of GALNT1 in HK-2 model showed reverse pattern compared with let-7i-5p, showing significantly reduced expression level following TGF- 31 treatment, which

Table 3. Post-hoc analysis of comparisons of parameters in HK2 cells shown in Figure 5A-C

\begin{tabular}{lccc}
\hline \multicolumn{4}{c}{ Tukey's multiple comparisons test } \\
\hline & \multicolumn{3}{c}{$p$ value } \\
\cline { 2 - 4 } & IL-6 & IL-1 $\beta$ & TNF- $\alpha$ \\
\hline CON $v$ s. TGF- $\beta 1$ & $<0.0001$ & $<0.0001$ & $<0.0001$ \\
CON $v$ s. TGF- $\beta 1+$ Let-Inh & 0.9377 & $>0.9999$ & 0.5919 \\
CON $v$ s. Let-mimic & $<0.0001$ & $<0.0001$ & $<0.0001$ \\
TGF- $\beta$ 1 vs. TGF- $\beta 1+$ Let-Inh & $<0.0001$ & $<0.0001$ & 0.0001 \\
TGF- $\beta 1$ vs. Let-mimic & 0.4865 & 0.8024 & 0.3951 \\
TGF- $\beta 1+$ Let-Inh $v s$. Let-mimic & $<0.0001$ & $<0.0001$ & $<0.0001$ \\
\hline
\end{tabular}

CON, control; Let-Inh, let-7i-5p inhibitor; Let-mimic, let-7i-5p mimic. was restored by miR inhibition (Fig. 4B). Level of GALNT1 was significant different among groups $(\mathrm{F}(4,10)=314.8$, $p<0.001)$ with detailed comparison between groups shown in Table 2. We then studied expression of Collagen I (COL1A1) and found that TGF-B1 significantly increased COL1A1 level which can be partially abolished by let-7i-5p inhibition (Fig. 4C). Level of COL1A1 was significant different among groups $(F(3,8)=668.1, p<0.001)$ with detailed comparison between groups shown in Table 2. At last, we found that protein level of GALNT1 showed corresponding pattern to the mRNA alteration (Fig. 4D). We then studied secretion of inflammatory cytokines in HK-2 cells. We found that TGF-ß1 induced, as expected, significantly elevated cytokine level which could be abolished by miR inhibition and mimicked by miR mimics (Fig. 5A). Level of IL-6 $(\mathrm{F}(3,8)=1461, p<0.001), \operatorname{IL}-1 \beta(\mathrm{F}(3,8)=339.7, p<0.001)$ and TNF- $\alpha(\mathrm{F}(3,8)=71.84, p<0.001)$ was significant different among groups, respectively, with detailed comparison between groups shown in Table 3. We also examined effect of GALNT1 overexpression (OE) and found that GALNT1OE abolished cytokine-inducing effect of let-7i-5p (Fig. 5B). Level of IL-6 $(\mathrm{F}(2,6)=69.63, p<0.001)$, IL-1 $\beta(\mathrm{F}(2,6)=$ 55.77, $p<0.001)$ and TNF- $\alpha(\mathrm{F}(2,6)=88.67, p<0.001)$ 
A

IL-6

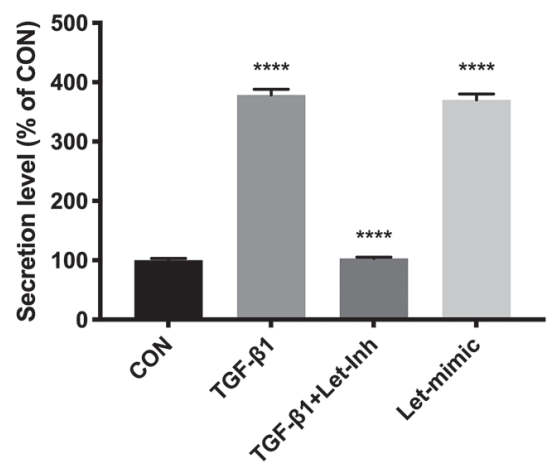

B

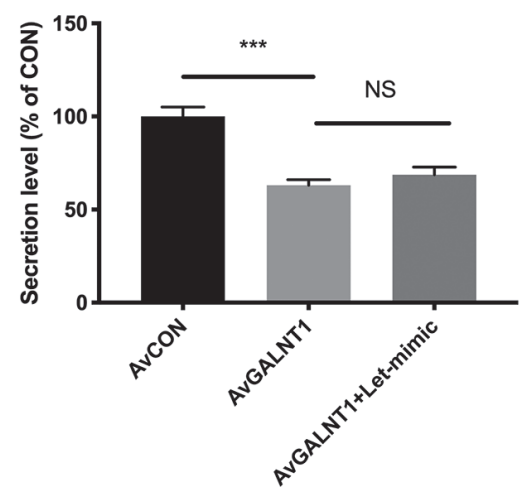

IL-1 $\beta$

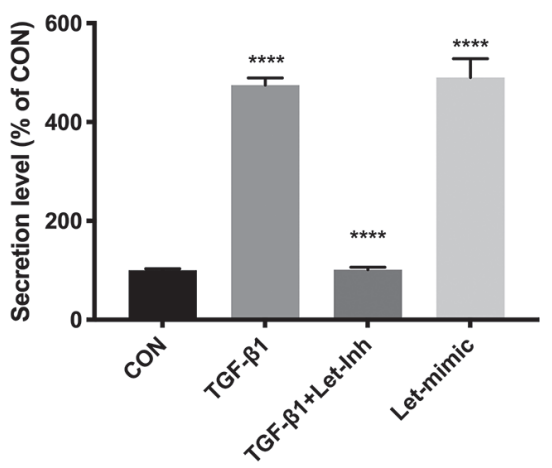

IL-1 $\beta$

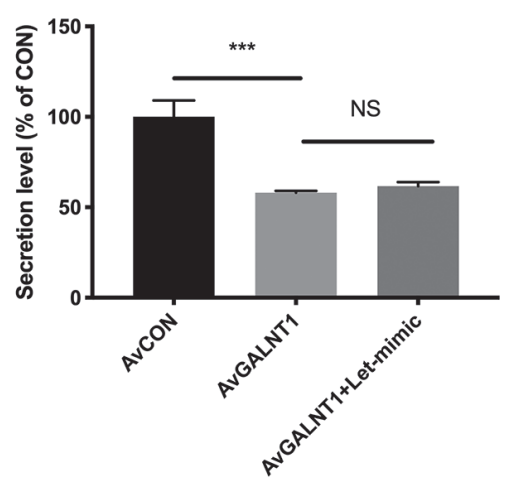

TNF- $\alpha$

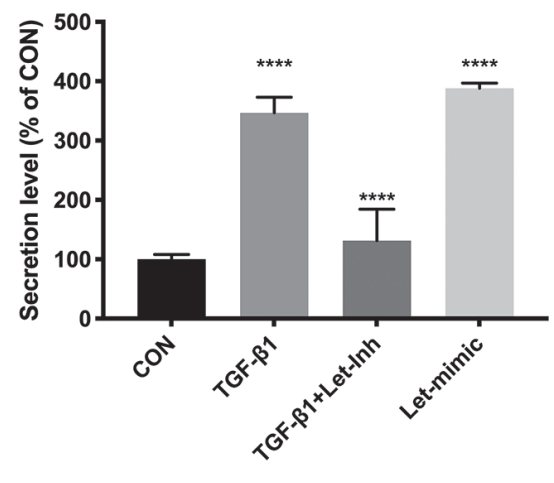

TNF- $\alpha$

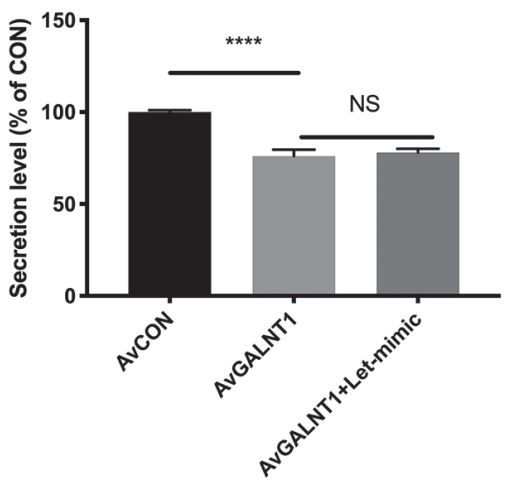

Figure 5. Overexpression of let-7i-5p inhibited secretion of IL-6, IL-1 $1 \beta$, and TNF- $\alpha$ in HK-2 cells treated with TGF- $\beta 1$ (A). Effect was abolished by simultaneous overexpression of GANLT1 and addition of let-7i-5p mimic (B). ${ }^{* * *} p<0.001,{ }^{* * *} p<0.0001$. CON, control; miRCon, control micro-RNA; Let-Inh, let-7i-5p inhibitor; Let-mimic, let-7i-5p mimic; AvCON, adenoviral control; AvGALNT1, adenoviral GALNT1; NS, not significant.

was significantly different among groups, respectively, with detailed comparison between groups shown in Table 4 .

\section{Discussion}

In the current study, we performed in silico analysis showing gradual increase of let-7i-5p and decrease of GALNT1 over time and the combination was validated both in mouse and human miR-gene target prediction databases. Compared with the sham group and negative group, expression of GALNT1 decreased while expression of let-7i-5p increased in renal tissues of both UUO and FA mice. Serum IL-6, IL-1 $\beta$, and TNF-a levels were elevated in vivo. In vitro models revealed negative correlation between expression levels of let-7i-5p and GALNT1. Overexpression of let-7i-5p in the cells inhibited GALNT1 expression. Though both genes were not reported in renal fibrosis, role of let-7i-5p in other pathophysiological processes that highly resemble renal fibrosis has been reported.

Let-7i-5p was reported to regulate cell morphology and migration through distinct signaling pathways in normal and pathogenic urethral fibroblasts (Zhang et al. 2020). They found that let-7i-5p regulates through its direct target genes involved in collagen metabolism, cell proliferation and differentiation, TGF-beta signaling, DNA repair and ubiquitination, gene silencing and oxygen homeostasis and concluded that let-7i-5p plays an essential role in regulating cell shape and tissue elasticity, cell migration, cell morphol-

Table 4. Post-hoc analysis of comparisons of parameters in HK2 cells shown in Figure 5D-F

\begin{tabular}{lccc}
\hline \multicolumn{4}{c}{ Tukey's multiple comparisons test } \\
\hline & \multicolumn{3}{c}{$p$ value } \\
\cline { 2 - 4 } & IL-6 & IL-1 $\beta$ & TNF- $\alpha$ \\
\hline AvCON vs. AvGALNT1 & $<0.0001$ & 0.0002 & $<0.0001$ \\
AvCON vs. AvGALNT1+Let-mimic & 0.0002 & 0.0003 & $<0.0001$ \\
AvGALNT1 vs. AvGALNT1+Let- & 0.2877 & 0.6984 & 0.6035 \\
mimic & & & \\
\hline
\end{tabular}

AvCON, adenoviral control; AvGALNT1, adenoviral GALNT1; Let-mimic, let-7i-5p mimic. 
ogy and cytoskeleton, and could serve as a potential target for clinical treatment of urethral stricture patients. In another report on pulmonary fibrosis, scholars found 2 lncRNAs, the MRAK088388 and MRAK081523, could regulate N4bp2 and Plxna4 expression by sponging miR-29b-3p and let-7i-5p, respectively, and possessed regulatory functions as ceRNAs.

GALNT1 encodes galactosamine:polypeptide N-acetylgalactosaminyltransferase (GalNAc-T) family of enzymes. GalNAc-Ts initiate mucin-type O-linked glycosylation in the Golgi apparatus by catalyzing the transfer of GalNAc to serine and threonine residues on target proteins. They are characterized by an N-terminal transmembrane domain, a stem region, a lumenal catalytic domain containing a GT1 motif and Gal/GalNAc transferase motif, and a C-terminal ricin/lectin-like domain. Though GANLT1 was not reported in fibrotic disease, its major biological effect, glycosylation plays critical roles in fibrosis. Zhang et al. (2019) reported that O-GlcNAc transferase suppresses necroptosis and liver fibrosis. They found that $\mathrm{O}$-linked $\beta$-N-acetylglucosamine (O-GlcNAc) modification protects against hepatocyte necroptosis and initiation of liver fibrosis. Wu et al. (2017) reported glycosylation-dependent galectin-1/ neuropilin-1 interactions promote liver fibrosis through activation of TGF- $\beta$ - and PDGF-like signals in hepatic stellate cells. They concluded that targeting Gal-1/NRP-1 interactions could be developed into liver fibrosis therapy. In another study by Moon, four novel biomarkers, including one glycosylation marker: ELF, M2BPGi, galectin-3, and sST2 were used in the comparison of liver fibrosis assessment. They concluded that although the biomarkers varied significantly according to TE grade, and each biomarker showed a different trend (Moon et al. 2018). ELF and M2BPGi seem to have comparable good performance for detecting liver fibrosis. Also, glycosylation also plays critical role in inflammation which is strongly associated with renal fibrosis. Alterations of glycosylation are observed in a number of inflammatory diseases. Pro-inflammatory cytokines have been shown to modulate cell surface glycosylation by regulating the expression of glycosyltransferases and sulfotransferases involved in the biosynthesis of glycan chains, inducing the expression of specific carbohydrate antigens at the cell surface that can be recognized by different types of lectins or by bacterial adhesins, contributing to the development of diseases (Groux-Degroote et al. 2020).

In conclusion, we showed here that dysregulation of let-7i-5p and GALNT1 play a role in renal fibrosis. Further investigation on GALNT1 functionality in renal fibrosis is warranted for its potential therapeutic effect.

Acknowledgements. This work was supported by the Foundation of Tongren Xinxing (No. 2019shtrxx07), Foundation of Science of Changning District (No.CNKW2018Y07), Sponsored by Shanghai Sailing Program (20YF1444000), and the Key projects of Science and Technology Committee of Changning District, Shanghai (CNKW2017Z01).

Conflict of interest. The authors declare no conflict of interest.

\section{References}

Groux-Degroote S, Cavdarli S, Uchimura K, Allain F, Delannoy P (2020): Glycosylation changes in inflammatory diseases (Review). Adv. Protein Hem. Struct. Biol. 119, 111-156 https://doi.org/10.1016/bs.apcsb.2019.08.008

Loboda A, Sobczak M, Jozkowicz A, Dulak J (2016): TGF-beta1/ Smads and miR-21 in renal fibrosis and inflammation. Mediators Inflamm. 2016, 8319283 https://doi.org/10.1155/2016/8319283

Lv W, Fan F, Wang Y, Gonzalez-Fernandez E, Wang C, Yang L, Booz GW, Roman RJ (2018): Therapeutic potential of microRNAs for the treatment of renal fibrosis and CKD. Physiol. Genomics 50, 20-34 https://doi.org/10.1152/physiolgenomics.00039.2017

Moon HW, Park M, Hur M, Kim H, Choe WH, Yun YM (2018): Usefulness of enhanced liver fibrosis, glycosylation isomer of Mac-2 binding protein, Galectin-3, and soluble suppression of tumorigenicity 2 for assessing liver fibrosis in chronic liver diseases. Ann. Lab. Med. 38, 331-337

https://doi.org/10.3343/alm.2018.38.4.331

Nastase MV, Zeng-Brouwers J, Wygrecka M, Schaefer L (2018): Targeting renal fibrosis: Mechanisms and drug delivery systems. Adv. Drug Deliv. Rev. 129, 295-307

https://doi.org/10.1016/j.addr.2017.12.019

Nogueira A, Pires MJ, Oliveira PA (2017): Pathophysiological mechanisms of renal fibrosis: A review of animal models and therapeutic strategies. In Vivo 31, 1-22 https://doi.org/10.21873/invivo.11019

Pavkovic M, Pantano L, Gerlach CV, Brutus S, Boswell SA, Everley RA, Shah JV, Sui SH, Vaidya VS (2019): Multi omics analysis of fibrotic kidneys in two mouse models. Sci. Data 6, 92 https://doi.org/10.1038/s41597-019-0095-5

Sun X, Liu Y, Li C, Wang X, Zhu R, Liu C, Liu H, Wang L, Ma R, Fu $\mathrm{M}$, et al. (2017): Recent advances of curcumin in the prevention and treatment of renal fibrosis. Biomed. Res. Int. 2017, 2418671 https://doi.org/10.1155/2017/2418671

Sun YB, Qu X, Caruana G, Li J (2016): The origin of renal fibroblasts/myofibroblasts and the signals that trigger fibrosis. Differentiation 92, 102-107 https://doi.org/10.1016/j.diff.2016.05.008

Wu MH, Chen YL, Lee KH, Chang CC, Cheng TM, Wu SY, Tu CC, Tsui WL (2017): Glycosylation-dependent galectin-1/neuropilin-1 interactions promote liver fibrosis through activation of TGF- $\beta$ - and PDGF-like signals in hepatic stellate cells. Sci. Rep. 7, 11006 https://doi.org/10.1038/s41598-017-11212-1

Zhang B, Li MD, Yin R, Liu Y, Yang Y, Mitchell-Richards KA, Nam HN, Li R, Wang L, Iwakari Y et al. (2019): O-GlcNAc transferase suppresses necroptosis and liver fibrosis. JCI Insight 4, e127709 https://doi.org/10.1172/jci.insight.127709

Zhang K, Yang R, Chen J, Qi E, Zhou S, Wang Y, Fu Q, Chen R, Fang $X(2020)$. Let-7i-5p regulation of cell morphology and migration through distinct signaling pathways in normal and pathogenic urethral fibroblasts. Front. Bioeng. Biotechnol. 8, 428 https://doi.org/10.3389/fbioe.2020.00428

Received: April 10, 2020

Final version accepted: January 11, 2021 